\title{
Cytotoxic clinical isolates of Pseudomonas aeruginosa identified during the Steroids for Corneal Ulcers Trial show elevated resistance to fluoroquinolones
}

\author{
Durga S Borkar ${ }^{1}$, Nisha R Acharya ${ }^{1,2,5}$, Chelsia Leong ${ }^{3}$, Prajna Lalitha ${ }^{4}$, Muthiah Srinivasan ${ }^{4}$, Catherine E Oldenburg ${ }^{2}$, \\ Vicky Cevallos ${ }^{2}$, Thomas M Lietman ${ }^{1,2,5}$, David J Evans ${ }^{3,6}$ and Suzanne M J Fleiszig ${ }^{3,7^{*}}$
}

\begin{abstract}
Background: To determine the relationship between type three secretion genotype and fluoroquinolone resistance for $P$. aeruginosa strains isolated from microbial keratitis during the Steroids for Corneal Ulcers Trial (SCUT) and for two laboratory strains, PA103 and PAO1.

Methods: Confirmed $P$. aeruginosa isolates from the SCUT were divided into exoU $(+)$ or exoU $(-)$. The exoU(+) strains contained the gene encoding ExoU, a powerful phospholipase toxin delivered into host cells by the type three secretion system. Isolates were then assessed for susceptibility to fluoroquinolone, cephalosporin, and aminoglycoside antibiotics using disk diffusion assays. Etest was used to determine the MIC of moxifloxacin and other fluoroquinolones. Laboratory isolates in which the exo $U$ gene was added or deleted were also tested.

Results: A significantly higher proportion of exoU $(+)$ strains were resistant to ciprofloxacin $(p=0.001)$, gatifloxacin $(p=0.003)$, and ofloxacin $(p=0.002)$ compared to exoU $(-)$ isolates. There was no significant difference between exoU(+) or exoU(-) negative isolates with respect to susceptibility to other antibiotics except gentamicin. Infections involving resistant exoU(+) strains trended towards worse clinical outcome. Deletion or acquisition of exoU in laboratory isolates did not affect fluoroquinolone susceptibility.

Conclusions: Fluoroquinolone susceptibility of $P$. aeruginosa isolated from the SCUT is consistent with previous studies showing elevated resistance involving exoU encoding (cytotoxic) strains, and suggest worse clinical outcome from infections involving resistant isolates. Determination of exo $\mathrm{U}$ expression in clinical isolates of P. aeruginosa may be helpful in directing clinical management of patients with microbial keratitis.
\end{abstract}

Keywords: $P$. aeruginosa, Microbial keratitis, SCUT, Fluoroquinolone resistance, ExoU

\section{Background}

Pseudomonas aeruginosa is a leading cause of bacterial keratitis involving contact lens wear or ocular injury [1-5]. While the pathogenesis of $P$. aeruginosa keratitis is complex, studies with in vivo models have shown that the type three secretion system (T3SS) is a significant contributor to disease pathogenesis [6-8]. Similar results

\footnotetext{
* Correspondence: fleiszig@berkeley.edu

${ }^{3}$ School of Optometry, University of California, 688 Minor Hall, 94720 Berkeley, CA, USA

'Programs in Vision Science, Infectious Diseases and Immunity, and Microbiology, University of California, 94720 Berkeley, CA, USA Full list of author information is available at the end of the article
}

have been obtained in murine models of acute pneumonia [9], and expression of the T3SS is associated with worse patient outcomes in ventilator-associated pneumonia and bacteremia $[10,11]$. Other clinical studies have also shown poor patient outcomes from $P$. aeruginosa infections are also associated with antimicrobial drug resistance $[12,13]$, a long recognized trait of this opportunistic pathogen [14].

The T3SS effector ExoU is a potent phospholipase that is cytotoxic towards host cells $[15,16]$. Several studies have shown that the exoU gene is selected for in clinical isolates from microbial keratitis [17-19], especially in

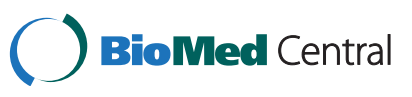


contact lens-associated cases [20]. Interestingly, several other studies have also shown a relationship between exo $U$ expression and resistance of $P$. aeruginosa to contact lens disinfectants [21], and to multiple antimicrobials [22], especially fluoroquinolones [23]. In the latter instance, it was shown that $P$. aeruginosa clinical isolates encoding exoU were more likely to encode multiple mutations in quinolone target genes, e.g. gyrA and parC, and suggested a co-evolution of these traits [24].

The Steroids for Corneal Ulcers Trial (SCUT), was a randomized controlled trial investigating the effects of adjunctive corticosteroid therapy to fluoroquinolone (moxifloxacin) treatment on the outcomes of bacterial keratitis [25,26]. Analysis of moxifloxacin resistance of bacterial keratitis isolates (including $P$, aeruginosa) from the SCUT study showed that increased resistance to this fluoroquinolone in vitro (i.e. increased minimum inhibitory concentration) was associated with poorer clinical outcomes, including reduced visual acuity at 3 weeks, a larger ulcer, and slower corneal re-epithelialization [27]. Here, we analyzed $P$. aeruginosa strains isolated from the SCUT study, and two laboratory isolates and their T3SS mutants, for their in vitro susceptibility to fluoroquinolones and other antimicrobials including cephalosporins and aminoglycosides. Antimicrobial classes and individual agents tested were chosen because of their common clinical empirical use against bacterial keratitis [28]. The objective was to determine if the SCUT clinical isolates and laboratory strains of $P$. aeruginosa also showed a relationship between fluoroquinolone resistance and exoU expression.

\section{Methods}

a) Clinical and laboratory isolates of P. aeruginosa and T3SS mutants

The $P$. aeruginosa isolates used were obtained from the Steroids for Corneal Ulcers Trial (SCUT), a randomized controlled trial investigating the effect of adjunctive corticosteroids on outcomes in bacterial keratitis [25]. The SCUT study was conducted with the approval of the University of California, San Francisco; Aravind Eye Hospital, Madurai, India; Dartmouth-Hitchcock Medical Center, Hanover, NH. Informed consent was obtained from all study participants. Corneal isolates were collected from patients with culture-confirmed bacterial keratitis. The isolates used in the present study were previously confirmed as $P$. aeruginosa using growth morphology, Gram stain, and API test strips, and their type three secretion gene profile was determined [29]. Presence of exoU was determined by polymerase chain reaction using primers and conditions described in detail by Ledbetter et al. [30]. Two wild-type laboratory strains of P. aeruginosa (PA103, encodes exoU) and PAO1 (does not encode exoU) were tested. For strain PA103, two T3SS

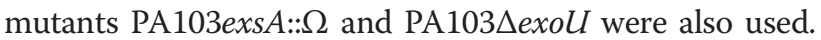
Strain PAO1 was complemented with a plasmid encoding exoU (pUCPexoU) or a plasmid control (pUCP18).

\section{b) Antibiotic susceptibility testing}

Susceptibility to moxifloxacin, the fluoroquinolone antibiotic used in the SCUT, was measured using the Etest method (AB BIODISK, Solna, Sweden) for all isolates using a standardized inoculum of $1 \times 10^{8} \mathrm{CFU} / \mathrm{mL}$ on Mueller-Hinton agar plates, which were then incubated at $35^{\circ} \mathrm{C}$. Disc diffusion susceptibility testing was performed for various other antibiotics (other fluoroquinolones, cephalosporins, and aminoglycosides) and $P$. aeruginosa strains classified as susceptible, intermediate, or resistant to each antibiotic tested based on the Clinical and Laboratory Standards Institute (CLSI) guidelines [31]. Since CLSI guidelines do not provide susceptibility ranges for the MIC of moxifloxacin for $P$. aeruginosa, susceptibility ranges for ciprofloxacin were used for Etest results. Laboratory personnel were masked as to $P$. aeruginosa strain identity. Laboratory isolates were tested after the conclusion of the SCUT using similar methods, which have been described previously [25]. An ATCC P. aeruginosa strain (ATCC 27853) was used for quality control when performing antibiotic susceptibility testing for laboratory strains.

\section{c) Visual acuity}

SCUT patients with culture-confirmed bacterial keratitis were evaluated at multiple time points, including enrollment and three months, by certified refractionists who performed visual acuity examinations. Visual acuity was measured as best spectacle-corrected visual acuity (BSCVA) in $\log$ MAR units, with $0.1 \log$ MAR corresponding to approximately one line of acuity. Laboratory personnel were masked to the clinical data.

\section{d) Statistical analysis}

Differences in disc diffusion susceptibility for various antibiotics, and mean minimum inhibitory concentration (MIC) of moxifloxacin, between exoU(+) or exoU(-) strain types were compared using Fisher's exact test and the Student's t-Test, respectively. The t-Test was also used to compare the change in BSCVA between enrollment and three months between patients with corneal ulcers caused by resistant or non-resistant exoU(+) P. aeruginosa.

\section{Results}

Of 500 patients enrolled in the SCUT, 110 had a corneal ulcer involving $P$. aeruginosa [32]. Confirmed $P$. aeruginosa isolates that were available for study were divided into two groups according to presence or absence of the exoU gene. Table 1 shows disc diffusion antimicrobial 
Table 1 Antibiotic susceptibility ${ }^{1}$ of SCUT Isolates of $P$. aeruginosa and T3SS genotype

\begin{tabular}{|c|c|c|c|c|c|}
\hline & \multicolumn{2}{|c|}{$\operatorname{exoU}(+)(n=19)$} & \multicolumn{2}{|c|}{$\operatorname{exoU}(-)(n=75)$} & \multirow[t]{2}{*}{$P$-value ${ }^{2}$} \\
\hline & Resistant & Non-resistant & Resistant & Non-resistant & \\
\hline \multicolumn{6}{|l|}{ Fluoroquinolones } \\
\hline Ciprofloxacin ${ }^{3}$ & $5(28 \%)$ & $13(72 \%)$ & $1(1 \%)$ & 74 (99\%) & 0.001 \\
\hline Gatifloxacin & $5(26 \%)$ & $14(74 \%)$ & $2(3 \%)$ & 73 (97\%) & 0.003 \\
\hline Ofloxacin & $6(32 \%)$ & $13(68 \%)$ & $3(4 \%)$ & 72 (96\%) & 0.002 \\
\hline \multicolumn{6}{|l|}{ Cephalosporins } \\
\hline Cefotaxime & $3(16 \%)$ & $16(84 \%)$ & $9(12 \%)$ & $66(88 \%)$ & 0.70 \\
\hline Ceftazidime & $3(16 \%)$ & $16(84 \%)$ & $5(7 \%)$ & 70 (93\%) & 0.20 \\
\hline \multicolumn{6}{|l|}{ Aminoglycosides } \\
\hline Amikacin & $1(5 \%)$ & $18(95 \%)$ & $1(1 \%)$ & 74 (99\%) & 0.37 \\
\hline Gentamicin & $5(26 \%)$ & $14(74 \%)$ & $4(5 \%)$ & 71 (95\%) & 0.02 \\
\hline Tobramycin & $3(16 \%)$ & $16(84 \%)$ & $2(3 \%)$ & 73 (97\%) & 0.054 \\
\hline
\end{tabular}

${ }^{1}$ Disc diffusion assay.

${ }^{2}$ Fisher's exact test.

${ }^{3}$ Disc diffusion susceptibility data for ciprofloxacin not available for one exoU(+) isolate $(n=18)$.

susceptibility data for $19 \operatorname{exo} U(+)$ and $75 \operatorname{exo} U(-)$ isolates. A significantly higher proportion of exo $U(+)$ strains were resistant to ciprofloxacin $(\mathrm{p}=0.001)$, gatifloxacin $(\mathrm{p}=0.003)$, and ofloxacin $(\mathrm{p}=0.002)$ compared to $\operatorname{exo} U(-)$ isolates. There was no significant difference between exoU $(+)$ or exou( $(-)$ negative isolates with respect to susceptibility to the cephalosporins tested, or to the aminoglycosides amikacin and tobramycin. Interestingly, there were significantly more gentamicin resistant $\operatorname{exo} U(+)$ isolates $(\mathrm{p}=0.02)$.

Of the $19 \operatorname{exoU}(+)$ corneal isolates, 4 were resistant to gatifloxacin, ciprofloxacin, and ofloxacin based on disc diffusion susceptibility testing, and had an MIC greater than or equal to 4 micrograms $/ \mathrm{ml}$. The BSCVA of patients with ulcers caused by the resistant ExoU(+) isolates improved, on average, approximately six lines less from enrollment compared to patients with ulcers caused by non-resistant $\operatorname{ExoU}(+)$ isolates $(-0.38 \log \mathrm{MAR}$ vs. -1.04 $\log M A R)$ although this difference was not statistically significant $(\mathrm{p}=0.08)$.

In another set of experiments, the MIC of moxifloxacin was determined and compared for 21 exoU(+) and $76 \operatorname{exoU}(-)$ SCUT isolates of $P$. aeruginosa. Figure 1 shows that isolates encoding exoU had, on average, a greater than two-fold higher MIC compared to exoU(-) strains $(\mathrm{p}=0.0001)$.

Laboratory isolates of $P$. aeruginosa were also tested to determine if genetic deletion of the T3SS, or deletion or acquisition of exoll, affected bacterial susceptibility to fluoroquinolones or other antibiotics using disc diffusion and Etest assays (Table 2). Neither deletion of the T3SS or exoU in the cytotoxic strain PA103, nor acquisition of exoU in the invasive strain PAO1, affected sensitivity to any antimicrobial tested.

\section{Discussion}

Topical fluoroquinolones are commonly used in the treatment of bacterial keratitis, in which $P$. aeruginosa is a leading causative pathogen. The data presented in this study show the antimicrobial resistance patterns of $P$. aeruginosa strains (94) isolated from patients during the

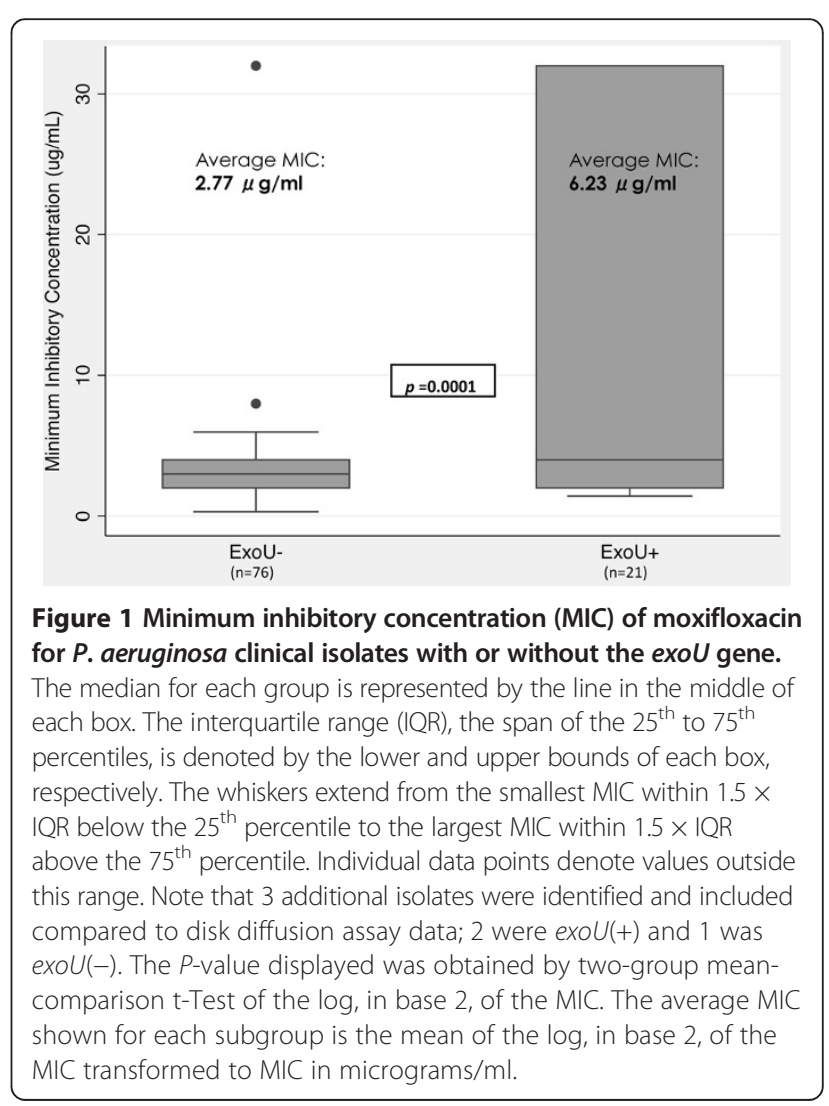


Table 2 Antibiotic susceptibility testing results for $\boldsymbol{P}$. aeruginosa laboratory isolates

\begin{tabular}{|c|c|c|c|c|c|c|c|c|}
\hline \multirow[b]{2}{*}{ Strain } & \multicolumn{7}{|c|}{ Disc diffusion susceptibility $^{1}$} & \multirow{2}{*}{$\begin{array}{l}\text { Etest }^{2} \\
\text { Moxi }\end{array}$} \\
\hline & Cipro & Gati & Oflox & Ceftaz & Gent & Tob & Moxi & \\
\hline PA103 & S & S & $\mathrm{s}$ & $\mathrm{S}$ & $\mathrm{s}$ & S & $\mathrm{S}$ & $S(1.0)$ \\
\hline PA103 exsA:: $\Omega$ & S & S & $S$ & S & $S$ & S & S & $S(0.5)$ \\
\hline PA103 $\Delta$ exoU & S & S & $\mathrm{s}$ & S & S & S & S & $S(0.5)$ \\
\hline PAO1 & S & S & S & S & S & s & S & $S(0.5)$ \\
\hline PAO1 + pUCP exOU & S & S & S & S & S & S & S & $S(0.5)$ \\
\hline $\mathrm{PAO} 1+\mathrm{pUCP} 18$ & S & S & S & S & S & S & S & $S(1.0)$ \\
\hline ATCC 27853 (QC) & s & s & S & s & $\mathrm{s}$ & s & 1 & I (2.0) \\
\hline
\end{tabular}

${ }^{1} \mathrm{~S}=$ Sensitive; I = Intermediate; R = Resistant. Ranges used to determine susceptibility were taken from the Clinical and Laboratory Standards Institute guidelines. QC $=$ Quality Control.

${ }^{2} \mathrm{~S}=$ Sensitive; I= Intermediate; $\mathrm{R}=$ Resistant. Numbers in parentheses represent the MIC (micrograms $/ \mathrm{ml}$ ).

SCUT. The data show a significant increase in the number of isolates resistant to fluoroquinolones if the bacteria encode the type three secreted cytotoxin ExoU. The data also confirm that deletion or acquisition of exoU gene does not affect fluoroquinolone susceptibility. These data are consistent with previous studies showing an association of exoU expression with fluoroquinolone resistance in $P$. aeruginosa, and support the hypothesis that T3SS expression and fluoroquinolone resistance are co-selected traits in both ocular and non-ocular clinical isolates [22,23]. Indeed, prior studies have found a significantly higher rate of gyrA mutations, combined with mutations in other fluoroquinolone target genes e.g. parC, in exoU encoding $P$. aeruginosa isolates [23,24].

It was of interest that we also found significantly more gentamicin resistant $\operatorname{exoU}(+)$ isolates in the study, although this did not occur for other aminoglycosides. This finding is consistent with a previous study looking at bacteremia isolates of $P$. aeruginosa in which the presence of exoU was also associated with increased resistance to gentamicin, but not amikacin [11]. It would be of interest to determine if gentamicin resistance in these ocular $\operatorname{exoU}(+)$ isolates relates to specific mutations in drug target genes, as shown for fluoroquinolones [24].

In bacterial keratitis, antibiotic susceptibility likely plays a role in clinical outcome, suggesting a role for testing antibiotic susceptibility in management decisions [27,33]. Interestingly, we found that SCUT patients with ulcers caused by fluoroquinolone resistant exou(+) P. aeruginosa isolates trended toward worse visual outcomes at three months compared to those with ulcers caused by non-resistant exoU(+) isolates. These results could reflect increased bacterial persistence in the cornea, allowing for greater tissue damage from bacterial toxins (including ExoU), and from host immune infiltrative responses.

The continuing emergence of antimicrobial resistant strains has the potential to influence "optimal" treatment of $P$. aeruginosa keratitis. However, $P$. aeruginosa resistance rates to fluoroquinolones and aminoglycosides, common choices for management of this condition, remain relatively low and stable in many places, although they can vary widely by geographical location and time [22,34-36]. Our study, and those of others [11,22-24], however, suggest that the combination of exoU and antibiotic resistance may be particularly deleterious to the patient and rapid identification of these isolates, if possible, could help optimize treatment.

\section{Conclusion}

Fluoroquinolone susceptibility of $P$. aeruginosa isolated from the SCUT is consistent with previous studies showing elevated resistance involving exoU encoding (cytotoxic) strains, and suggest worse clinical outcome from infections involving these resistant isolates. Increased gentamicin resistance is also associated with exoU(+) strains. Determination of exoU expression in clinical isolates of $P$. aeruginosa, and antibiotic susceptibility testing, may be helpful in directing optimal clinical management of patients with microbial keratitis if methods could be developed with sufficient speed and accuracy, and which could be applied in a clinical setting.

\section{Competing interests}

Dr. Suzanne Fleiszig is a paid consultant for Allergan, Irvine, CA. That work is unrelated to the content of this manuscript. There are no competing

interests for any of the authors.

\section{Authors' contributions}

Study design and analysis: DB, SF, NA, DE, PL, MS, TL. Experiments: DB, CL, CO, VC. Manuscript writing: DB, DE, NA, SF. Supervision: SF, NA, TL. All authors read and approved the final manuscript.

\section{Acknowledgements}

We thank the SCUT data and safety monitoring board: Marian Fisher, Ph.D. (chair), Anthony Aldave, M.D., Donald Everett, M.A., Jacqueline Glover, Ph.D., K. Ananda Kannan, M.D., Steven Kymes, Ph.D., G.V.S. Murthy, M.D., and Ivan Schwab, M.D. The Steroids for Corneal Ulcers Trial was funded by National Eye Institute grant U10 EY015114. Dr. Acharya is supported by a National Eye 
Institute K23EY017897 grant and a Research to Prevent Blindness Career Development Award. Dr. Fleiszig is supported by National Institutes of Health grants EY011221 and Al079192. The UCSF Department of Ophthalmology is supported by a National Eye Institute core grant, EY02162, an unrestricted grant from Research to Prevent Blindness, the South Asia Research Fund, and That Man May See, Inc.

\section{Author details}

'Department of Ophthalmology, University of California, 94143 San Francisco, CA, USA. ${ }^{2}$ Francis I. Proctor Foundation, University of California, 94143 San Francisco, CA, USA. ${ }^{3}$ School of Optometry, University of California, 688 Minor Hall, 94720 Berkeley, CA, USA. ${ }^{4}$ Aravind Eye Care System, Madurai, Tamil Nadu, India. ${ }^{5}$ Department of Epidemiology and Biostatistics, University of California, 94143 San Francisco, CA, USA. ${ }^{6}$ College of Pharmacy, Touro University California, 94592 Vallejo, CA, USA. ${ }^{7}$ Programs in Vision Science, Infectious Diseases and Immunity, and Microbiology, University of California, 94720 Berkeley, CA, USA.

Received: 20 December 2013 Accepted: 11 April 2014

Published: 24 April 2014

\section{References}

1. Stapleton F, Carnt N: Contact lens-related microbial keratitis: how have epidemiology and genetics helped us with pathogenesis and prophylaxis. Eye (Lond) 2012, 26(2):185-193.

2. Stapleton F, Keay LJ, Sanfilippo PG, Katiyar S, Edwards KP, Naduvilath T: Relationship between climate, disease severity, and causative organism for contact lens-associated microbial keratitis in Australia. Am J Ophthalmol 2007, 144(5):690-698.

3. Lin CC, Lalitha P, Srinivasan M, Prajna NV, McLeod SD, Acharya NR, Lietman TM, Porco TC: Seasonal trends of microbial keratitis in South India. Cornea 2012, 31(10):1123-1127.

4. Laspina F, Samudio M, Cibils D, Ta CN, Farina N, Sanabria R, Klauss V, Mino de Kaspar H: Epidemiological characteristics of microbiological results on patients with infectious corneal ulcers: a 13-year survey in Paraguay. Graefes Arch Clin Exp Ophthalmol 2004, 242(3):204-209.

5. Green M, Apel A, Stapleton F: Risk factors and causative organisms in microbial keratitis. Cornea 2008, 27(1):22-27.

6. Lee EJ, Cowell BA, Evans DJ, Fleiszig SM: Contribution of ExsA-regulated factors to corneal infection by cytotoxic and invasive Pseudomonas aeruginosa in a murine scarification model. Invest Ophthalmol Vis Sci 2003, 44(9):3892-3898.

7. Lee EJ, Evans DJ, Fleiszig SM: Role of Pseudomonas aeruginosa ExsA in penetration through corneal epithelium in a novel in vivo model. Invest Ophthalmol Vis Sci 2003, 44(12):5220-5227.

8. Zolfaghar I, Evans DJ, Ronaghi R, Fleiszig SM: Type III secretion-dependent modulation of innate immunity as one of multiple factors regulated by Pseudomonas aeruginosa RetS. Infect Immun 2006, 74(7):3880-3889.

9. Shaver CM, Hauser AR: Relative contributions of Pseudomonas aeruginosa ExoU, ExoS, and ExoT to virulence in the lung. Infect Immun 2004, 72(12):6969-6977.

10. Hauser AR, Cobb E, Bodi M, Mariscal D, Valles J, Engel JN, Rello J: Type III protein secretion is associated with poor clinical outcomes in patients with ventilator-associated pneumonia caused by Pseudomonas aeruginosa. Crit Care Med 2002, 30(3):521-528.

11. El-Solh AA, Hattemer A, Hauser AR, Alhajhusain A, Vora H: Clinical outcomes of type III Pseudomonas aeruginosa bacteremia. Crit Care Med 2012, 40(4):1157-1163.

12. Bradbury R, Champion A, Reid DW: Poor clinical outcomes associated with a multi-drug resistant clonal strain of Pseudomonas aeruginosa in the Tasmanian cystic fibrosis population. Respirology 2008, 13(6):886-892.

13. Hsu DI, Okamoto MP, Murthy R, Wong-Beringer A: Fluoroquinolone-resistant Pseudomonas aeruginosa: risk factors for acquisition and impact on outcomes. J Antimicrob Chemother 2005, 55(4):535-541.

14. Garau J, Gomez L: Pseudomonas aeruginosa pneumonia. Curr Opin Infect Dis 2003, 16(2):135-143.

15. Finck-Barbancon V, Goranson J, Zhu L, Sawa T, Wiener-Kronish JP, Fleiszig SM, Wu C, Mende-Mueller L, Frank DW: ExoU expression by Pseudomonas aeruginosa correlates with acute cytotoxicity and epithelial injury. Mol Microbiol 1997, 25(3):547-557.
16. Sato H, Frank DW, Hillard CJ, Feix JB, Pankhaniya RR, Moriyama K, FinckBarbancon V, Buchaklian A, Lei M, Long RM, Wiener-Kronish J, Sawa T: The mechanism of action of the Pseudomonas aeruginosa-encoded type III cytotoxin. ExoU EMBO J 2003, 22(12):2959-2969.

17. Lomholt JA, Poulsen K, Kilian M: Epidemic population structure of Pseudomonas aeruginosa: evidence for a clone that is pathogenic to the eye and that has a distinct combination of virulence factors. Infect Immun 2001, 69(10):6284-6295.

18. Winstanley C, Kaye SB, Neal TJ, Chilton HJ, Miksch S, Hart CA: Genotypic and phenotypic characteristics of Pseudomonas aeruginosa isolates associated with ulcerative keratitis. J Med Microbiol 2005, 54(Pt 6):519-526.

19. Stewart RM, Wiehlmann $L$, Ashelford KE, Preston SJ, Frimmersdorf $E$, Campbell BJ, Neal TJ, Hall N, Tuft S, Kaye SB, Winstanley C: Genetic characterization indicates that a specific subpopulation of Pseudomonas aeruginosa is associated with keratitis infections. J Clin Microbiol 2011, 49(3):993-1003.

20. Choy MH, Stapleton F, Willcox MD, Zhu H: Comparison of virulence factors in Pseudomonas aeruginosa strains isolated from contact lens- and noncontact lens-related keratitis. J Med Microbio/ 2008, 57(Pt 12):1539-1546.

21. Lakkis C, Fleiszig SM: Resistance of Pseudomonas aeruginosa isolates to hydrogel contact lens disinfection correlates with cytotoxic activity. J Clin Microbiol 2001, 39(4):1477-1486.

22. Zhu H, Conibear TC, Bandara R, Aliwarga Y, Stapleton F, Willcox MD: Type III secretion system-associated toxins, proteases, serotypes, and antibiotic resistance of Pseudomonas aeruginosa isolates associated with keratitis. Curr Eye Res 2006, 31(4):297-306.

23. Wong-Beringer A, Wiener-Kronish J, Lynch S, Flanagan J: Comparison of type III secretion system virulence among fluoroquinolone-susceptible and -resistant clinical isolates of Pseudomonas aeruginosa. Clin Microbiol Infect 2008, 14(4):330-336.

24. Agnello M, Wong-Beringer A: Differentiation in Quinolone Resistance by Virulence Genotype in Pseudomonas aeruginosa. PLoS One 2012, 7(8):e42973.

25. Srinivasan M, Mascarenhas J, Rajaraman R, Ravindran M, Lalitha P, Glidden DV, Ray KJ, Hong KC, Oldenburg CE, Lee SM, Zegans ME, McLeod SD, Lietman TM, Acharya NR: The Steroids for Corneal Ulcers Trial: Study Design and Baseline Characteristics. Arch Ophthalmol 2012, 130(2):151-157.

26. Srinivasan M, Mascarenhas J, Rajaraman R, Ravindran M, Lalitha P, Glidden DV, Ray KJ, Hong KC, Oldenburg CE, Lee SM, Zegans ME, McLeod SD, Lietman TM, Acharya NR: Corticosteroids for bacterial keratitis: the Steroids for Corneal Ulcers Trial (SCUT). Arch Ophthalmol 2012, 130(2):143-150.

27. Lalitha P, Srinivasan M, Manikandan P, Bharathi MJ, Rajaraman R, Ravindran M, Cevallos V, Oldenburg CE, Ray KJ, Toutain-Kidd CM, Glidden DV, Zegans ME, McLeod SD, Acharya NR, Lietman TM: Relationship of in vitro susceptibility to moxifloxacin and in vivo clinical outcome in bacterial keratitis. Clin Infect Dis 2012, 54(10):1381-1387.

28. Hanet MS, Jamart J, Chaves AP: Fluoroquinolones or fortified antibiotics for treating bacterial keratitis: systematic review and meta-analysis of comparative studies. Can J Ophthalmol 2012, 47(6):493-499.

29. Borkar DS, Fleiszig SM, Leong C, Lalitha P, Srinivasan M, Ghanekar AA, Tam C, Li WY, Zegans ME, McLeod SD, Lietman TM, Acharya NR: Association between cytotoxic and invasive Pseudomonas aeruginosa and clinical outcomes in bacterial keratitis. JAMA Ophthalmol 2013, 131(2):147-153.

30. Ledbetter EC, Mun JJ, Kowbel D, Fleiszig SM: Pathogenic phenotype and genotype of Pseudomonas aeruginosa isolates from spontaneous canine ocular infections. Invest Ophthalmol Vis Sci 2009, 50(2):729-736.

31. National Committee for Clinical Laboratory Standards (NCCLS): Performance standards for antimicrobial susceptibility testing: tenth informational supplement M100-S10 (M2). In Wayne, PA: NCCLS

32. Sy A, Srinivasan M, Mascarenhas J, Lalitha P, Rajaraman R, Ravindran M, Oldenburg CE, Ray KJ, Glidden D, Zegans ME, McLeod SD, Lietman TM, Acharya NR: Pseudomonas aeruginosa keratitis: outcomes and response to corticosteroid treatment. Invest Ophthalmol Vis Sci 2012, 53(1):267-272.

33. Chen A, Prajna L, Srinivasan M, Mahalakshmi R, Whitcher JP, McLeod S, Lietman TM, Acharya NR: Does in vitro susceptibility predict clinical outcome in bacterial keratitis? Am J Ophthalmol 2008, 145(3):409-412.

34. Orlans $\mathrm{HO}$, Hornby SJ, Bowler IC: In vitro antibiotic susceptibility patterns of bacterial keratitis isolates in Oxford, UK: a 10-year review. Eye (Lond) 2011, 25(4):489-493. 
35. Willcox MD: Review of resistance of ocular isolates of Pseudomonas aeruginosa and staphylococci from keratitis to ciprofloxacin, gentamicin and cephalosporins. Clin Exp Optom 2011, 94(2):161-168.

36. Smitha S, Lalitha P, Prajna VN, Srinivasan M: Susceptibility trends of Pseudomonas species from corneal ulcers. Indian J Med Microbiol 2005, 23(3):168-171.

doi:10.1186/1471-2415-14-54

Cite this article as: Borkar et al: Cytotoxic clinical isolates of

Pseudomonas aeruginosa identified during the Steroids for Corneal

Ulcers Trial show elevated resistance to fluoroquinolones. BMC

Ophthalmology 2014 14:54.

\section{Submit your next manuscript to BioMed Central and take full advantage of:}

- Convenient online submission

- Thorough peer review

- No space constraints or color figure charges

- Immediate publication on acceptance

- Inclusion in PubMed, CAS, Scopus and Google Scholar

- Research which is freely available for redistribution 